\title{
Segmentação de Texturas Monocromáticas usando Transformada Wavelet Packet
}

\author{
Karinne S. Silva e Yuzo Iano
}

\begin{abstract}
Resumo-Este trabalho propõe uma nova metodologia nãosupervisionada para segmentação de imagens de textura monocromáticas. As características utilizadas para agrupar os pixels são obtidas através da Transformada Wavelet Packet. Para realizar a clusterização são utilizados dois algoritmos: ARIA e k-means. O primeiro tem a função de inicializar os valores dos protótipos dos clusters, cabendo ao segundo finalizar a segmentação. Dessa forma é possível diminuir as desvantagens da inicialização aleatória e da determinação do número de agrupamentos existentes na implementação padrão do k-means, sem elevar significativamente o custo computacional.
\end{abstract}

Palavras-Chave-Análise de textura, segmentação de textura, wavelet packet.

Abstract-This work proposes a new unsupervised methodology for monochromatic texture images segmentation. The characteristics utilized for grouping the pixels are obtained by wavelet packet transform. To perform the clustering two algorithms are used: ARIA and k-means. The first one has the function of initialize the values of the prototypes of the clusters, falling to the second finalize the segmentation. Thus it is possible to reduce the disadvantages of the random initialization and of the determination of the number of existing groups in the implementation standard of the k-means, without elevate significantly the computational cost.

Keywords-Texture analysis, texture segmentation, wavelet packet.

\section{INTRODUÇÃO}

Texturas contêm informações importantes para o ser humano e são bastante utilizadas na interpretação e análise de diversos tipos de superfícies e objetos, podendo ser definidas como um conjunto de relações espaciais e arranjos de elementos básicos em uma vizinhança.

A análise textural é uma tarefa difícil na área de processamento de imagens. Porém, esta é uma etapa fundamental em muitas aplicações, tais como sensoriamento remoto, diagnóstico médico, reconhecimento de padrões e inspeção de superfícies.

Podem ser encontrados na literatura diversos métodos para análise de textura [1], [2], [3]. Segundo Livens [4] podemos dividi-los em 4 segmentos:

- Estatísticos: Em geral, estes métodos conseguem maiores taxas de discriminação do que os métodos estruturais e as transformações. Exemplos desse tipo de método são a análise de Histograma [5], Matriz de Co-ocorrência [6] e o espectro de textura;

Karinne S. Silva e Yuzo Iano, Departamento de Comunicações, Faculdade de Engenharia Elétrica e de Computação, Universidade Estadual de Campinas, Campinas, Brasil, E-mails: karinne@decom.fee.unicamp.br, yuzo@decom.fee.unicamp.br. Este trabalho foi parcialmente financiado pela CAPES.
- Estruturais: Nestes métodos, a textura é definida como uma composição de padrões primitivos organizados de acordo com algumas regras. Têm a vantagem de produzir uma boa descrição da imagem, porém são mais úteis para formá-la (síntese) do que realizar propriamente a análise. Um exemplo de ferramenta para análise estrutural é a morfologia matemática [7];

- Modelados: Realizam a análise utilizando modelos matemáticos (fractais e estocásticos). Alguns parâmetros da imagem são extraídos do modelo e atuam como características para analisar a imagem. Sua desvantagem é a complexidade computacional na estimação dos parâmetros [8];

- Transformações: Neste caso, a imagem é representada em um novo espaço, por exemplo, os espaços de freqüência e escala, onde as características texturais se tornam mais acessíveis. Os principais exemplos são as transformadas de imagens através de Fourier, Gabor [9] e Wavelet [10].

Recentemente, métodos baseados em Transformada Wavelet [11], [12], têm sido extremamente explorados por proverem a caracterização de uma imagem em diferentes escalas. Neste trabalho, será utilizada uma abordagem particular da Transformada Wavelet, denominada Transformada Wavelet Packet (TWP), que tem sido muito utilizada principalmente em problemas de classificação de textura.

A metodologia descrita neste trabalho utiliza a TWP para a extração das características dos pixels da imagem. Em seguida dois algoritmos, ARIA e k-means, são utilizados em conjunto para realizar o agrupamento dos pixels e a segmentação da imagem.

Na próxima seção faremos uma revisão sobre Transformada Wavelet e decomposição por wavelet packet. A seção III apresenta o método utilizado para obtenção das características de textura através dos coeficientes da decomposição. A metodologia proposta para realizar a segmentação de imagens a partir das características obtidas será detalhada na seção IV. Na seção V são apresentados os resultados experimentais obtidos com o método proposto. Finalmente, na seção VI encontram-se expostas as conclusões e perspectivas para trabalhos futuros.

\section{Transformada WAVElet PaCKet}

\section{A. Transformada Wavelet}

O objetivo principal do uso da Transformada Wavelet é representar um sinal como uma superposição de funções de base ortogonal, chamadas wavelets. Estas são obtidas através de operações de dilatação e translação de uma única função chamada wavelet-mãe, representada na Equação 1: 


$$
\psi_{m, n}=2^{-m / 2} \psi\left(2^{-m} x-n\right)
$$

onde $m$ e $n$ são inteiros. Os coeficientes wavelet resultantes da decomposição de um sinal qualquer $f(x)$ podem ser obtidos utilizando a Equação 2.

$$
c_{m, n}=\int_{-\infty}^{\infty} f(x) \psi_{m, n}(x) d x
$$

e, dados os coeficientes $c_{m, n}$, pode-se obter o sinal original $f(x)$ pela Equação 3.

$$
f(x)=\sum_{m, n} c_{m, n} \psi_{m, n}(x)
$$

A wavelet-mãe $\psi(x)$ é construída a partir de uma função de escalonamento $\phi(x)$ de acordo com as Equações 4 e 5 [9].

$$
\begin{aligned}
& \psi(x)=\sqrt{2} \sum_{k} g(k) \phi(2 x-k) \\
& \phi(x)=\sqrt{2} \sum_{k} h(k) \phi(2 x-k)
\end{aligned}
$$

onde

$$
g(k)=(-1)^{k} h(1-k) .
$$

Os coeficientes $h(k)$ na Equação 5 devem cumprir uma série de restrições para que a base de funções wavelet seja ortonormal [12]. Podem ser encontrados na literatura vários conjuntos desses coeficientes, alguns dos mais conhecidos e utilizados foram propostos por Daubechies em [13].

Temos que $h(x)$ e $g(x)$ representam filtros passabaixa e passa-alta, respectivamente. Eles são utilizados na decomposição por Transformada Wavelet para gerar as imagens de aproximação e de detalhes. Para a obtenção dos vários de níveis de decomposição, a imagem de aproximação é decomposta novamente em imagem de aproximação e de detalhes. Os coeficientes de aproximação e de detalhes são, em geral, utilizados na obtenção das características necessárias para classificação e segmentação de imagens.

A imagem de textura D56 retirada do álbum de Brodatz [14] e sua decomposição por Transformada Wavelet de nível 2 podem vistas na Figura 1.

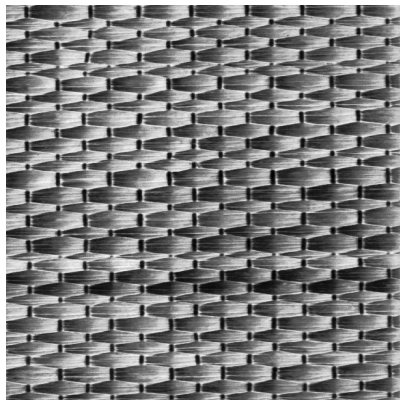

(a)

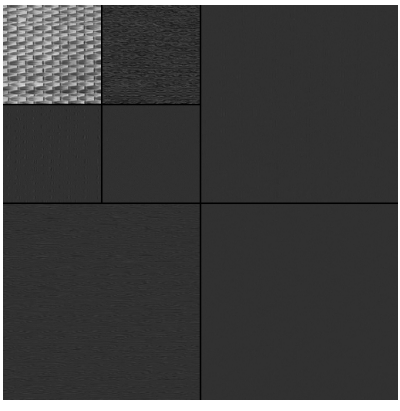

(b)
Fig. 1. (a) Imagem original. (b) Transformada Wavelet de nível 2.

\section{B. Wavelet Packet}

Segundo Zhang [11], a decomposição por wavelet packet é uma generalização da decomposição wavelet clássica. Assim como no método de Fourier, qualquer função $f(x) \in L^{2}(\Re)$ pode ser decomposta em funções wavelet packet.

A decomposição por wavelet packet é capaz de decompor o sinal também em frequiências médias, e não apenas em baixas e altas. Esta propriedade é de fundamental importância para a caracterização da textura, pois há muitas informações texturais presentes na faixa de freqüência intermediária [12].

$\mathrm{O}$ conjunto de funções wavelet packet $\left\{W_{n}\right\}_{n=0}^{\infty}$ pode ser gerado a partir de uma dada função $W_{0}$ :

$$
\begin{gathered}
W_{2 n}(x)=\sqrt{2} \sum_{k} h(k) W_{n}(2 x-k) \\
W_{2 n+1}(x)=\sqrt{2} \sum_{k} g(k) W_{n}(2 x-k)
\end{gathered}
$$

onde $W_{0}$ e $W_{1}$ são similares à função de escalonamento $\phi$ e à wavelet-mãe $\psi$ da Transformada Wavelet, respectivamente [12].

A principal diferença entre a decomposição por wavelet packet e a Transformada Wavelet tradicional consiste em que, no caso da primeira, as decomposições não são aplicadas somente ao componente de baixa freqüência, mas ao resultado obtido na saída de todos os filtros.

A Figura 2 ilustra a decomposição por wavelet packet sob forma de árvore [15], onde $S$ é o sinal original, $D$ a imagem de detalhes e $A$ a imagem de aproximação. Maiores detalhes sobre a TWP podem ser encontrados em [12], [15] e [16].

A Figura 3 mostra a decomposição da imagem D56 por wavelet packet. Na Figura 3.(b) podemos observar os coeficientes da decomposição por wavelet packet no nível 2. Vemos que as imagens de detalhes que tinham sido obtidas no nível 1 também foram subdivididas gerando 16 componentes e não 7, como na Transformada Wavelet original.

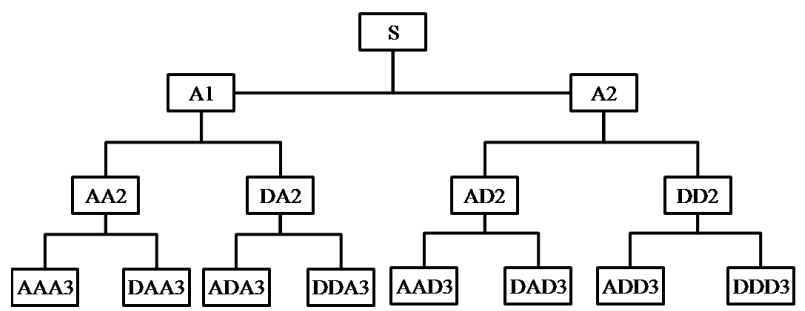

Fig. 2. Trasformada Wavelet Packet.

\section{EXTRAÇÃo DE CARACTERÍSTICAS UTILIZANDO WAVELET PACKET}

Inicialmente, as imagens são decompostas através da Transformada Wavelet Packet, apresentada na seção II, sendo obtidas quatro sub-imagens. Apenas os coeficientes de detalhes (componentes vertical, horizontal e diagonal) serão utilizados como características, para que não tenhamos influência das informações de tons de cinza presente na sub-imagem de baixa freqüência. 


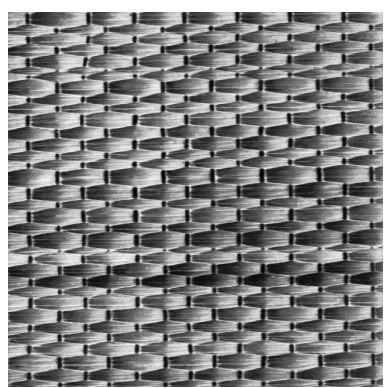

(a)

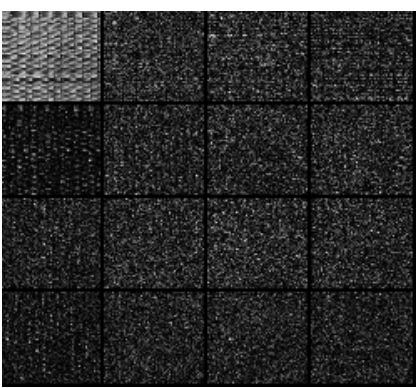

(b)
Fig. 3. (a) Imagem original. (b) Transformada Wavelet Packet de nível 2.

Em seguida, a energia de cada informação é calculada para cada pixel, de acordo com a Equação 9.

$$
E_{x, y}^{B}=f^{B}(x, y)^{2}
$$

onde $B$ representa cada uma das sub-imagens e $f^{B}(x, y)$ seu valor no pixel $(x, y)$.

Para cada pixel serão obtidas três características que, antes de serem utilizadas para segmentação da imagem, passarão por um processo de normalização, posicionando os valores no intervalo $[0,255]$.

Com o intuito de escolher apenas as características mais relevantes, foi utilizada a Transformada de Karhunen-Loève, também conhecida por análise de componentes principais [17], que reduzirá o número de características para as duas mais significativas, evitando que informações não relevantes para a clusterização aumentem o tempo necessário para o processamento.

\section{Segmentação De TeXtura}

Após a extração de características, para obter a segmentação da imagem, é necessário o agrupamento dos pixels que apresentam as mesmas características texturais.

Para realizar essa tarefa foi escolhido o algoritmo k-means [18], bastante difundido na literatura. Este algoritmo fornece um agrupamento de informações de acordo com os próprios dados, com base em análises e comparações entre os seus valores numéricos, fornecendo uma clusterização automática, ou seja, não supervisionada. Sua execução começa com a definição do valor inicial dos protótipos dos clusters, onde o resultado final é altamente dependente dessa inicialização.

Existem duas desvantagens na execução básica do k-means. A primeira está no fato dele não ser capaz de determinar automaticamente o número de clusters presentes no agrupamento, geralmente fornecido pelo usuário. Em seguida, sua inicialização padrão é aleatória, o que pode gerar resultados diferentes cada vez que ele é executado. Isto força o usuário a realizar inúmeras execuções para escolher aquela que tiver o melhor resultado.

Com o objetivo de minimizar estes dois problemas, neste trabalho, foi utilizado outro algoritmo, chamado ARIA (Adaptive Radius Immune Algorithm) [19]. Sua função consiste em realizar uma pré-clusterização, fornecendo ao k-means o valor dos protótipos iniciais dos clusters. Dessa forma, obtemos um resultado final com o mínimo de variação entre execuções.
O ARIA faz parte de um conjunto de algoritmos imunoinspirados, assim como o aiNet [20]. Neste tipo de algoritmo, os dados a serem classificados são denominados antígenos e os protótipos dos agrupamentos, anticorpos. Os principais diferenciais do ARIA em relação aos outros algoritmos de clusterização estão na capacidade de determinar automaticamente o número de agrupamentos e de considerar informações de densidade dos dados. O pseudo-código do ARIA está representado no Algoritmo 1 abaixo.

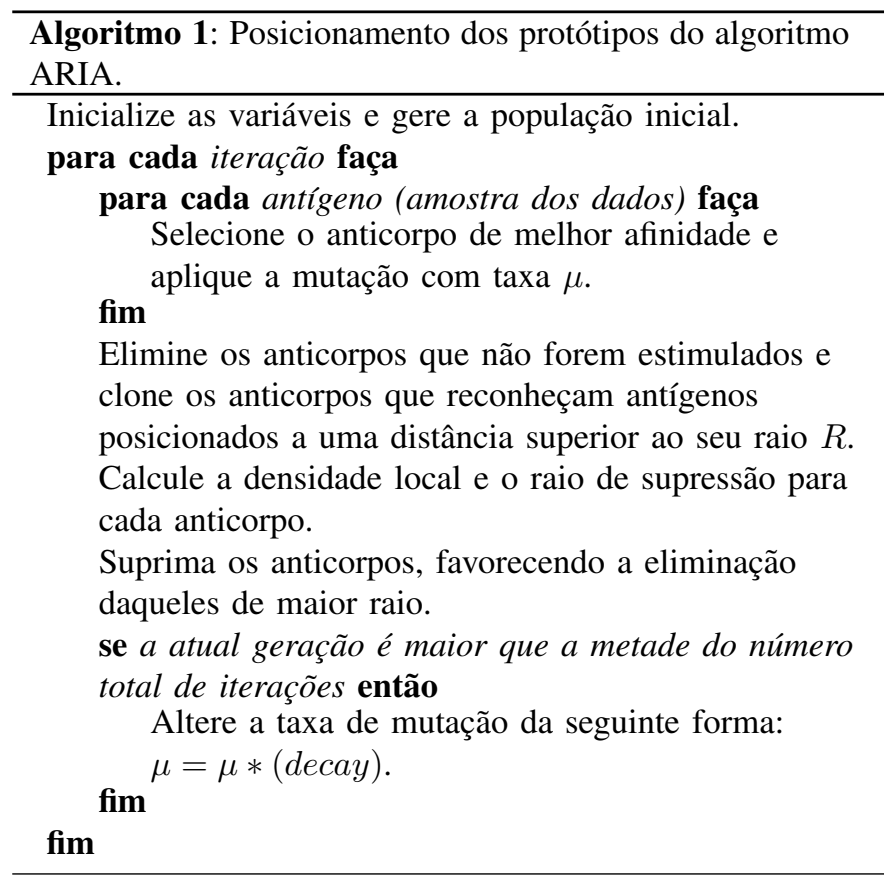

A descrição completa do algoritmo ARIA e maiores detalhamentos sobre os mecanismos empregados podem ser encontrados em [19].

Após utilizarmos o ARIA para localizar os protótipos iniciais dos clusters, empregamos o k-means para formar os agrupamentos de pixels, realizando a segmentação. Foi aplicado o filtro da mediana, com tamanho de janela $7 \times 7$ ao resultado final da segmentação.

\section{RESUlTAdOS EXPERIMENTAIS}

Esta seção apresenta os resultados dos testes realizados com a metodologia proposta.

A Figura 4.(a) mostra uma imagem de tamanho 157x200 pixels composta pelas texturas D49 (fundo) e D53 (padrão interno) da coleção de Brodatz. Ela foi utilizada por Jenssen e Eltoft [21], que apresenta uma metodologia denominada ICA (Independent Component Analysis) e por Nunes e Conci [22], onde é apresentado um novo coeficiente utilizado na segmentação de texturas, chamado CVE (Coeficiente de Variação Espacial).

Observa-se que resultado obtido pelo método proposto é superior aquele apresentado por Jenssen. Todavia, uma pequena queda na qualidade da segmentação é observada em relação à obtida por Nunes e Conci [22], porém este último utiliza um método supervisionado, onde o usuário escolhe amostras das texturas para serem utilizados como conjunto de treinamento. 


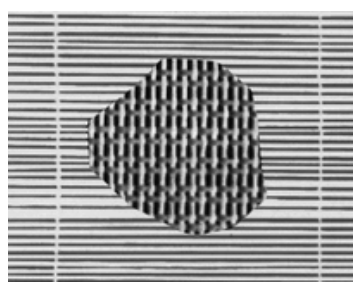

(a)

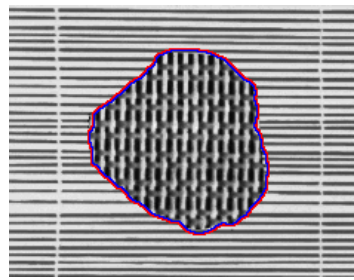

(c)

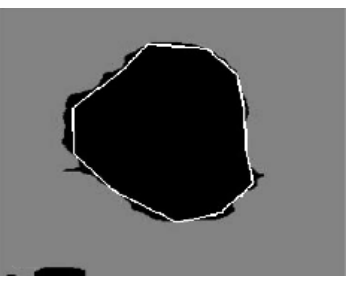

(b)

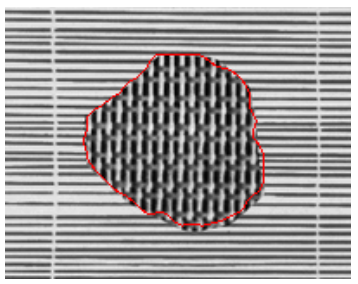

(d)
Fig. 4. (a) Imagem original. (b) Resultado obtido por Jenssen (cor preta) e o contorno desejado em branco. (c) Resultado obtido por Nunes (contorno colorido). (d) Resultado obtido pela metodologia proposta (contorno em vermelho).

A Figura 5.(a) apresenta uma imagem monocromática de tamanho 480x192 pixels, que representa as letras UCSB em segmentos de linhas diagonais. Essa imagem é usada como exemplo pelo grupo JSEG, encontrando-se disponível no site do grupo [23] e por Nunes e Conci [22].

A Figura 5.(b) mostra o resultado obtido pelo grupo JSEG, a Figura 5.(c) apresenta o resultado obtido com a implementação do CVE e na Figura 5.(d) encontra-se o resultado obtido com a metodologia proposta. Verifica-se que os contornos foram bem localizados pelos 3 métodos.

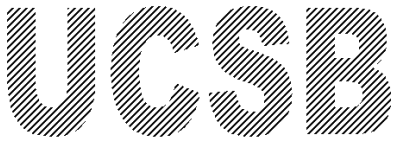

(a)

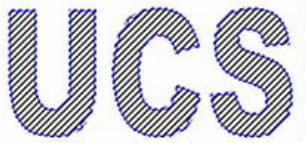

(c)

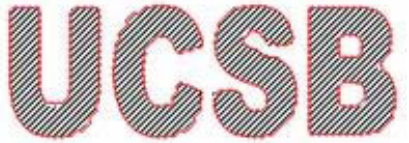

(b)

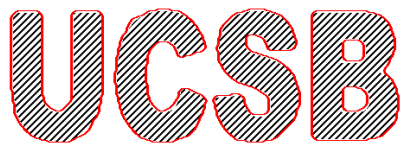

(d)
Fig. 5. (a) Imagem original. (b) Resultado obtido por Jenssen. (c) Resultado obtido por Nunes. (d) Resultado obtido pela metodologia proposta.

A Figura 6 mostra o teste realizado com uma imagem montada com as texturas D78 (fundo) e D56 (quadrado) do álbum de Brodatz.

A Figura 6.(b) mostra o melhor resultado obtido com a aplicação do método proposto sem a aplicação do ARIA para inicializar o k-means. Neste caso a inicialização dos protótipos é feita de forma aleatória e o usuário determina o número de clusters. As Figuras 6.(c) e 6.(d) apresentam os resultados obtidos pela metodologia proposta.

Verifica-se que a segmentação realizada utilizando o ARIA para inicialização do k-means obteve melhor resultado final.

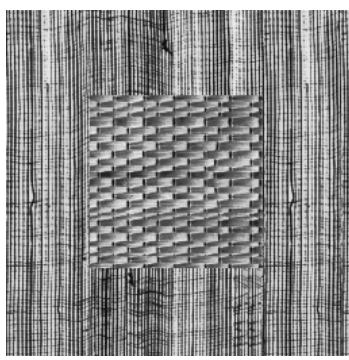

(a)

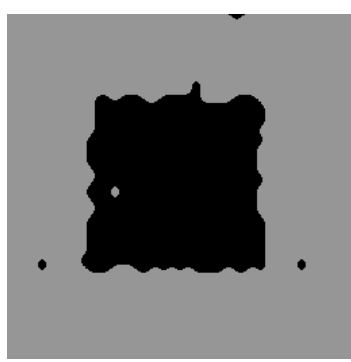

(c)

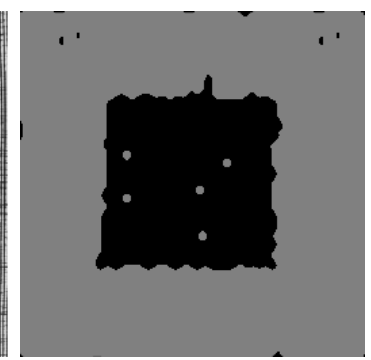

(b)

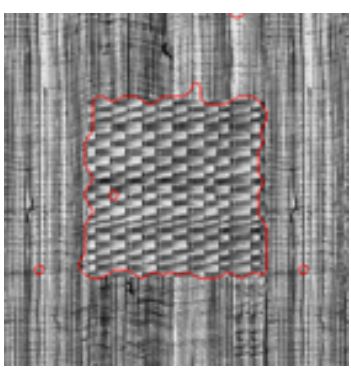

(d)
Fig. 6. (a) Imagem original. (b) Resultado obtido pelo k-means. (c) Resultado obtido pelo método proposto. (d) Resultado obtido pelo método proposto sobreposto à imagem original (contorno vermelho).

\section{Conclusões}

Neste trabalho foi apresentada uma nova metodologia nãosupervisionada para segmentação de imagens de textura. Os principais diferenciais aqui apresentados são a utilização da TWP como extrator de características, sendo capaz de obter informações de média freqüência, e do algoritmo ARIA para inicialização do k-means, reduzindo os problemas causados pela inicialização aleatória e determinando automaticamente o número de clusters.

Foram apresentados resultados experimentais que comprovam a eficiência do método. Nas imagens utilizadas nos testes foi possível distinguir os padrões de texturas existentes na imagem. Foram feitas várias comparações com outros métodos existentes na literatura, em algumas comparações o resultado obtido pelo nosso método foi superior, em outras, o resultado foi similar, tendo o nosso método o diferencial de não necessitar da interferência do usuário.

Vemos aqui um potencial para expandir este trabalho, obtendo a segmentação de imagens com mais de dois padrões de texturas e para imagens coloridas. Para isso temos como objetivos: eliminar totalmente o k-means do processo de segmentação e realizar a decomposição por wavelet packet em mais níveis, aumentando o número de características de textura, possibilitando uma melhor distinção entre os diversos padrões.

\section{AGRADECIMENTOS}

Aos órgãos FAPESP (Fundação de Amparo à Pesquisa do Estado de São Paulo), FAEPEX (Fundo de Apoio ao Ensino, à Pesquisa e à Extensão), CNPq (Conselho Nacional de Desenvolvimento Científico e Tecnológico) e CAPES (Coordenação de Aperfeiçoamento de Pessoal de Nível Superior) e ao Programa CAPES de Formação de Recursos Humanos em 
Televisão Digital (RH-TVD) pelo apoio às pesquisas desenvolvidas no Laboratório de Comunicações Visuais (LCV) da Unicamp.

\section{REFERÊNCIAS}

[1] R.M. Haralick, "Statistical and structural approaches to texture," Proceedings of the IEEE, v. 67, n. 5, pp. 786-804, 1979.

[2] N. Ahuja e A. Rosenfeld, "Mosaic Models for Textures," IEEE Transactions on Pattern Analysis and Machine Intelligence, v. PAMI-3, n. 1, pp. 1-11, 1981.

[3] F. S. Cohen, Z. Fan e M. A. Patel, "Classification of rotated and scaled textured images using Gaussian Markov random field models," IEEE Transactions on Pattern Analysis and Machine Intelligence, v. 13, n. 2, pp. 192-202, 1991.

[4] S. Livens, Image analysis for material characterization, Ph.D. Dissertation, Antwerpen University, 1998.

[5] X. Liu e D. Wang, "Texture classification using spectral histograms," IEEE Transactions on Image Processing, v. 12, n. 6, pp. 661$670,2003$.

[6] M. Hauta-Kasari, J. Parkkinen, T. Jaaskelainen, e R. Lenz, "Generalized co-occurrence matrix for multispectral texture analysis," Proceedings of the 13th International Conference on Pattern Recognition, v. 2, pp. 785789, 1996.

[7] J. Sobus, B. Pourdeyhimi, B. Xu e Y. Ulcay, "Evaluating Loss of Texture Definition in Carpets Using Mathematical Morphology: Covariance," Textile Research Journal, v. 62, n. 1, pp. 26-39, 1992.

[8] W. H. Nailon, S. McLaughlin, T. Spencer, M. P. Ramo, D. M. Salter, G. R. Sutherland, E. A. Fox e W. N. McDicken, "Fractal texture analysis: an aid to tissue characterisation with intravascular ultrasound," Engineering in Medicine and Biology Society, 1997. Proceedings of the 19th Annual International Conference of the IEEE, v.2, pp. 534-537, 1997.

[9] A. Teuner, O. Pichler, e B.J. Hosticka, "Unsupervised texture segmentation of images using tuned matched Gabor filters", IEEE Transactions on Image Processing, v. 4, n. 6, pp. 863-870, 1995.

[10] M. Unser, "Texture classification and segmentation using wavelet frames," IEEE Transactions on Image Processing, v. 4, n. 11, pp. 15491560, 1995.

[11] Y. Zhang, X.-J. He e J.-H. Han, "Texture Feature-Based Image Classification Using Wavelet Package Transform," Lecture Notes In Computer Science, v. 3644, pp. 165-173, 2005.

[12] T. Chang e C.-C. J. Kuo, "Texture Analysis and Classification with TreeStructured Wavelet Transform," IEEE Transaction on Image Processing, v. 2, n. 4, pp. 429-441, 1993.

[13] I. Daubechies, "Orthonormal bases of compactly supported wavelets,"Communications on Pure and Applied Mathematics, v. 41, pp. 909-996, 1988.

[14] T. Randen, Brodatz Textures. Disponível em: http://www.ux.uis.no/ tranden/brodatz.html

[15] A. K. Soman e P. P. Vaidyanathan, "On orthonormal wavelets and paraunitary filter banks," IEEE Transactions on Signal Processing, v. 41, n. 3, pp. 1170-1183, 1993

[16] A. Laine e J. Fan, "Texture classification by wavelet packet signatures," IEEE Transactions on Pattern Analysis and Machine Intelligence, v. 15, n. 11 , pp. $1186-1191,1993$.

[17] S. Wold, K. Esbensen e P. Geladi, "Principal component analysis," Chemometrics and Intelligent Laboratory Systems. v. 2, n. 1-3, pp. 37-52, 1987.

[18] G. B. Coleman e H. C. Andrews, "Image segmentation by clustering," Proceedings of the IEEE, v. 67, n. 5, pp. 773-785, 1979.

[19] G. B. Bezerra, T. V. Barra, L. N. de Castro e F. J. Von Zuben, "Adaptive radius immune algorithm for data clustering," Lecture Notes on Computer Science, v. 3627, pp. 290-303, 2005.

[20] L. N. de Castro e F. J. Von Zuben, "aiNet: An artificial immune network for data analysis,"In H. A. Abbass, R. A. Sarker, e C. S. Newton (Eds.), Data Mining: A Heuristic Approach, pp. 231-259, USA: Idea Group Publishing, 2001.

[21] R. Jenssen e T.Eltoft, "Independent Component Analysis for Texture Segmentation," Pattern Recognition, v. 36, n. 10, pp. 2301-2315, 2003.

[22] E. O. Nunes e A. Conci, "Segmentação por Textura e Localização do Contorno de Regiões em Imagens Multibandas," IEEE Latin America Transactions, v. 5, n. 3, pp. 185-192, 2007.

[23] JSEG - Segmentation of Color-Texture Regions in Images and Video, Disponível em: http://vision.ece.ucsb.edu/segmentation/jseg/jsegIllusionary.html 